\title{
DESIGN AND SIMULATION OF RADIO FREQUENCY SEEKER ANTENNA STABILIZATION
}

\author{
M Geetha Reddy ${ }^{1}$, R. Sundararajan ${ }^{2}$ \\ ${ }^{\text {I} P o s t g r a d u a t e ~ S t u d e n t, ~ D i g i t a l ~ E l e c t r o n i c s ~ \& ~ C o m m u n i c a t i o n ~ S y s t e m s, ~ J I E T, ~ E N G I N E E R, ~ B F L, ~ H y d e r a b a d, ~ I n d i a ~}$ \\ ${ }^{2}$ Scientist-G (Retd.), DRDO \& Research Director, BFL, Hyderabad, India
}

\begin{abstract}
Present day guided weapon systems, especially tactical class missiles use RF seeker, for target tracking towards terminal engagement. The seeker system including its antenna assembly will be onboard the missile. Due to the missile trajectory corrections, the seeker antenna pointing to the target may get disturbed resulting in track loss. To avoid this track loss, it becomes necessary to stabilize the antenna system in two planes. The fundamental role of stabilization loop in seeker application is to precisely follow the angular rate of the target. In order to achieve this requirement, it is essential to highly isolate the gimbaled antenna from the missile body motion due to the maneuvering of target or low frequency vibration during flight. However, the isolation ratio and stability margin of stabilization loop adopting the gimbaled platform with both low stiffness and heavy inertia are limited by mechanical characteristic such as low resonance frequency and its high magnitude. The selection of proper feedback sensors, modeling of the total system are key features of this project. In the end, the performance and the stability of designed stabilization loop are demonstrated using simulation in both frequency and time domain. The Hardware for the system is under realization by the Industry. The whole scheme is simulated in MATLAB off-line for this project.
\end{abstract}

Keywords: Missile, RF seeker, Track loss, Stabilization loop, Angular Rate Command, Bore-Sight Error, Maneuvering and Gimbaled Platform.

\section{INTRODUCTION}

Different Guidance schemes are available to guide the missiles towards intended target for tactical missile systems. For this function, the target needs to be tracked continuously with respect to time. Active homing Guidance is one of the scheme used in tactical missiles during the final phase of engagement. Though various schemes are available for mechanizing homing guidance [2], [3], the current technology is to use active guidance where a RF seeker is used to measure LOS rotation rate as well as closing velocity, between target \& missile. The output from the seeker will be used by guidance system to generate command which is executed by Flight control system. Due to this trajectory correction process the antenna may get disturbed from the track. In other words, the track loss will occur.

This antenna stabilization system will be realized for operation in Real-time system which is being developed. The objective of the project is to design and generate specifications for the subsystems of the servo which has to meet the following requirements:

1. Servo should follow the command input (Target following).

2. Stabilization scheme should reject the disturbance due to trajectory correction executed by the missile in shortest possible time, i.e., output of servo system should fall to zero as early as possible for the disturbance input.
When the commands generated are used for driving the missile towards target, there exists a possibility of seeker antenna losing the track of target, resulting in large miss distance between Missile \& Target [1]. To avoid losing the track in spite of missile motion, it becomes necessary to stabilize the antenna by proper servo loop, here by providing good degree of isolation between missile body \& Antenna system.

The Servo loop achieve this isolation has to be made much faster than the tracking loop and hence results in high gain system. This high gain loop design will provide adequate stiffness.

This Paper deals with the design of such antenna stabilization loop, which tracks the continuously moving target \& places the antenna on the target quickly, whenever it gets disturbed due to missile motion.

In other words the servo loop ensures 'No Track loss'.

\section{SPECIFICATIONS FOR SERVO DESIGN}

- Stabilization Band width: $30 \pm 5 \mathrm{~Hz}$

- Body disturbance max :100 deg/Sec

- Body acceleration Max:1000 deg/ $\mathrm{S}^{2}$

- Target angular rate : 0.5 to $30 \mathrm{deg} / \mathrm{Sec}$

- Gimbal angular freedom : $\pm 40^{\circ}$

- Isolation/Decoupling High

- Accuracy : $0.4 \mathrm{deg}$

- Bandwidth : $2 \mathrm{~Hz}$ 
- $\quad$ Load Diameter : $240 \mathrm{~mm}$ (typical)

- Antenna Weight: $750 \mathrm{~g}$

\subsection{Analysis of Specifications}

As per specifications the following parameters are calculated

Inertia of the load $(\mathrm{Ja})=6.48 * 10^{-3} \mathrm{Kg}-\mathrm{m}^{2}$

Torque of the motor $(\mathrm{Tm})=0.113 \mathrm{~N}-\mathrm{m}$

A suitable motoris selected to overcome the inertia and accelerate the motor.

\section{RESPONSE OF LOAD}

Load is the high inertia Load. The response of the load in the open loop condition is studied for step, ramp and acceleration inputs. The plant responses are deviating, which necessitate adoption of closed loop control. The responses of the system are as in figure 1.
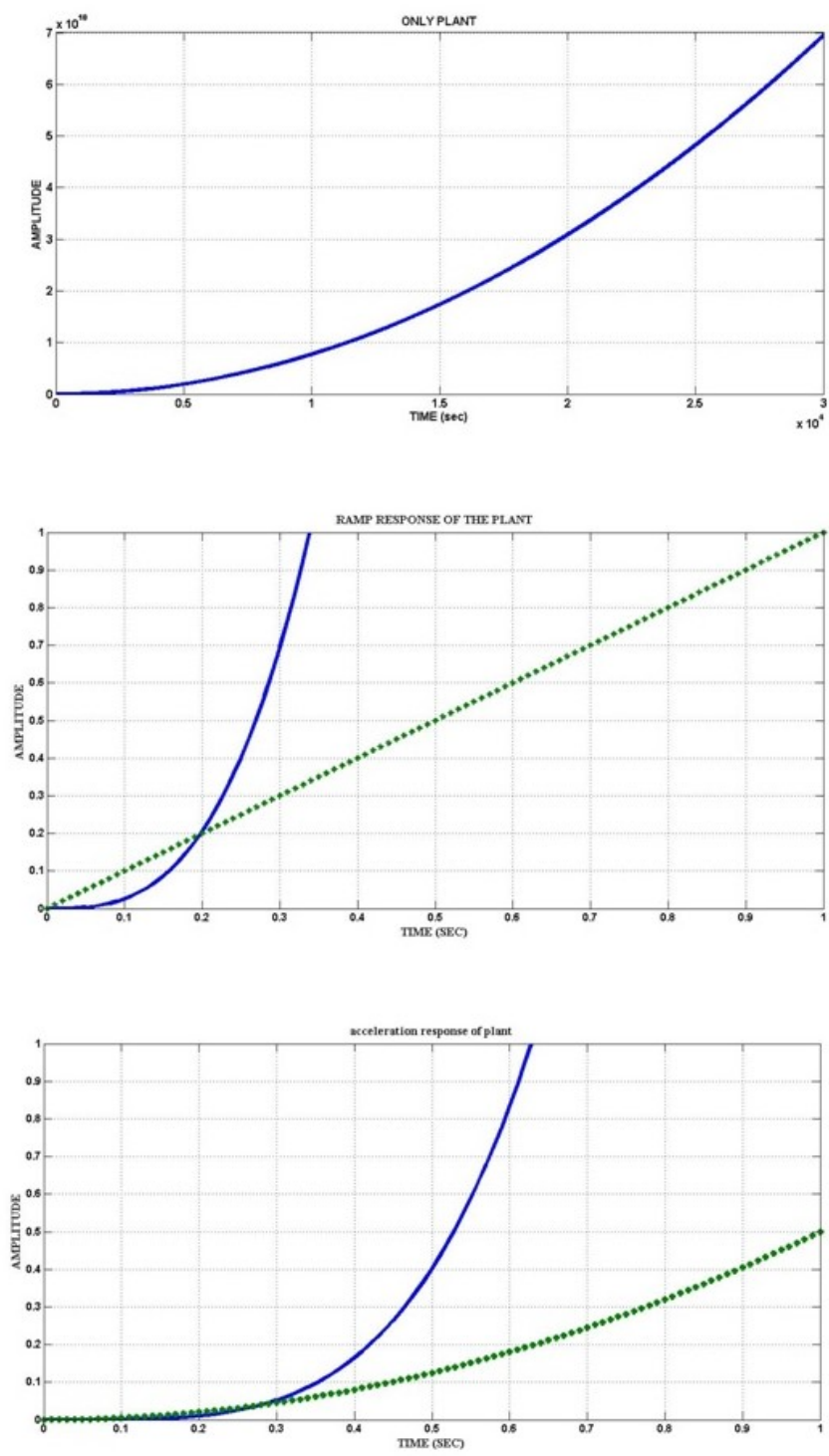

Fig 1: Open Loop Response of Plant

\section{CLOSED LOOP SYSTEM RESPONSE}

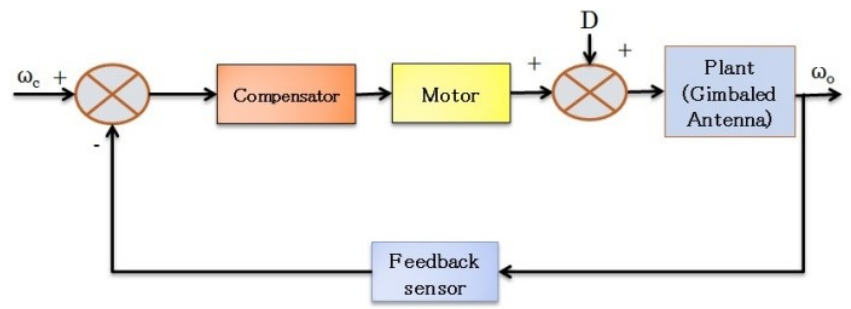

Fig 2: Closed Loop System

Above Figure 2 represents the closed loop system, where $\omega_{\mathrm{c}}$ is the command in $\mathrm{rad} / \mathrm{s}, \omega_{0}$ is output rate $\mathrm{rad} / \mathrm{sec}, \mathrm{D}$ is the disturbance torque acting on the antenna assembly.

The motor transfer function relating the angular rate to input excitation voltage is mathematically derived and numerical values for various coefficients of the transfer function are taken from the selected motor catalog and given as below

Transfer function of the motor between rate and input voltage:

$$
\frac{\omega}{v}=\frac{2.415 \mathrm{e} 007}{s^{2}+1250 s+434800}
$$

Where $\omega$ is angular rate of motor in $\mathrm{rad} / \mathrm{sec}$ and $v$ is the voltage input.

The loop analysis is done in two cases.

Case 1: without compensator i.e., with simple gain the response of the system is as shown in the below Figure 3.

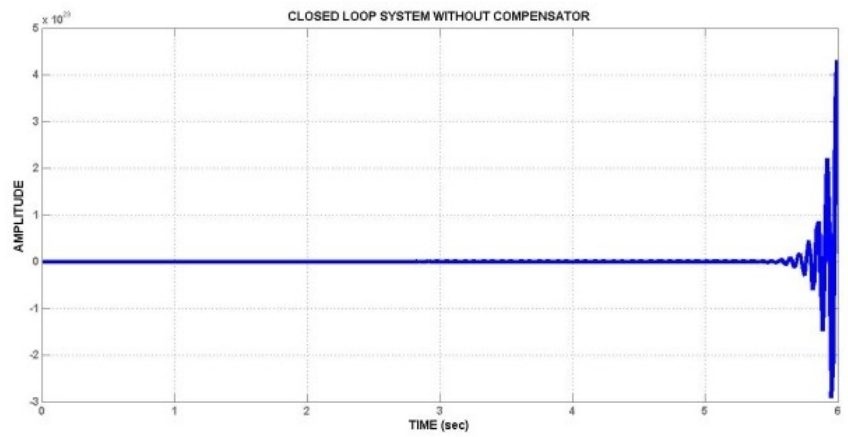

Fig 3: Closed Loop system response without compensator

From Figure 3 we can observe that the system response is unstable. So a suitable compensator has to be designed.

Case 2: To improve the transient response a Phase Lead compensator was designed using Bode method and the Response of the closed loop system was studied.

The Response of the closed loop system with the above Phase Lead Compensator is as shown in Figure 4 


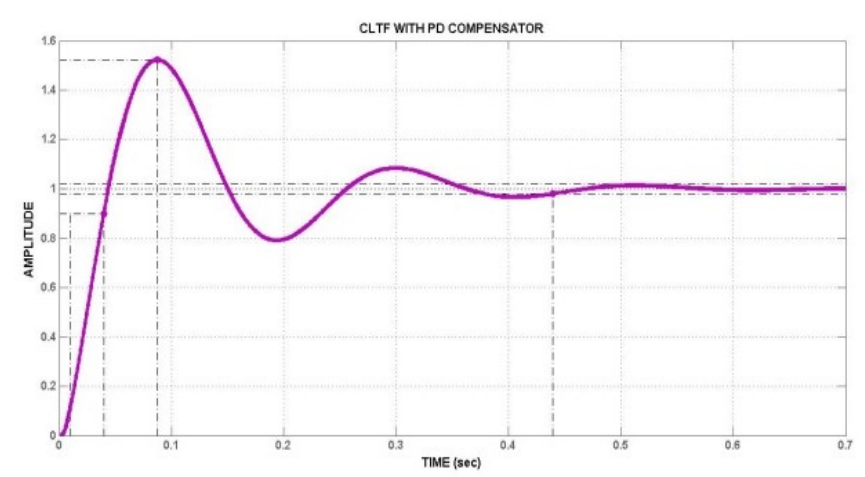

Fig 4: Closed Loop system response with a Phase Lead Compensator

The analysis of the above plot indicates the overshoot of $52 \%$ and settling time of $0.44 \mathrm{sec}$. This overshoot is not acceptable from performance point of view. Hence the zero location of the compensator has been varied and finally positioned at $\mathrm{s}=-5$ with this the response obtained is as shown in Figure 5.

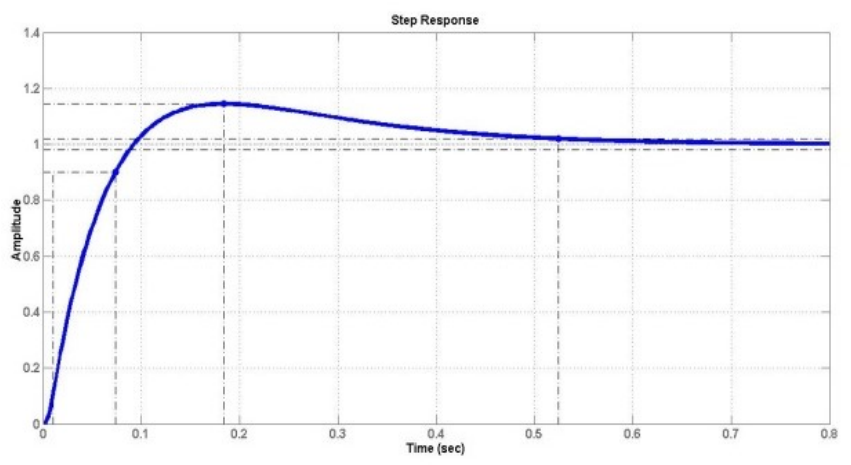

Fig 5: Closed Loop system response with a Phase Lead Compensator zero located at $\mathrm{s}=-5$.

Analysis of the result indicates the improvement of overshoot to $14 \%$ which is satisfactory for the system. Settling time is not much changed by this modification.

The results have been verified by Bode method for both uncompensated and compensated system. The stability margins obtained for both the systems are as given below

Table 1: Margins of the system

\begin{tabular}{|l|l|l|}
\hline Parameters & $\underline{\text { Uncompensated }}$ & Compensated \\
\hline Gain margin & $0 \mathrm{~dB}$ & $24.45 \mathrm{~dB}$ \\
\hline Phase margin & $-15.0726^{0}$ & $70.26^{0}$ \\
\hline
\end{tabular}

The corresponding bode plots are given in Figure 6
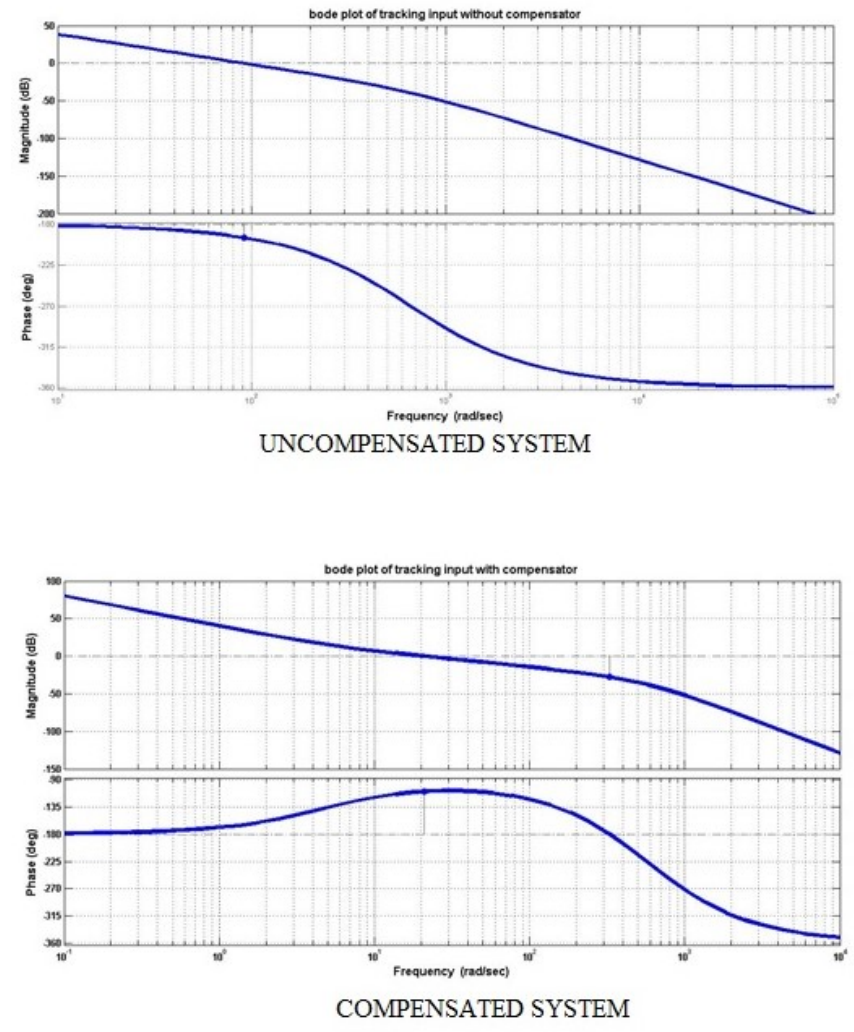

Fig 6: Bode Plots of uncompensated and compensated system.

\section{PARAMETRIC STUDY}

The feedback sensor to be utilized in the actual system is the Rate Gyro feedback. This offers good damping to the total response. The undamped natural frequency of the gyro which will be selected for such application will be greater than $120 \mathrm{~Hz}$. This is very far away from the frequency region of interest of the servo loop. Hence the dynamics of the sensor can be neglected and only the steady state gain of the sensor can be used for loop gain analysis. This gain has been varied from 0.2 to 1 and the response studied. It has been found that around 0.8 to 0.9 sufficient loop gain is present in the system to offer the required stiffness.

\section{SIMULATION OF THE TOTAL CONTROL LOOP}

The whole loop simulation is performed in MATLAB \& SIMULINK for three conditions [4]

1. Normal Tracking.

2. Disturbance Rejection.

3. Tracking and Disturbance simultaneously acting.

It may be noted that Sl.No. 3 is the realistic condition which will occur during the flight. Sl.No $1 \& 2$ are for the performance evaluation under individual conditions.

The Simulink simulation setup is as given in Figure 7 


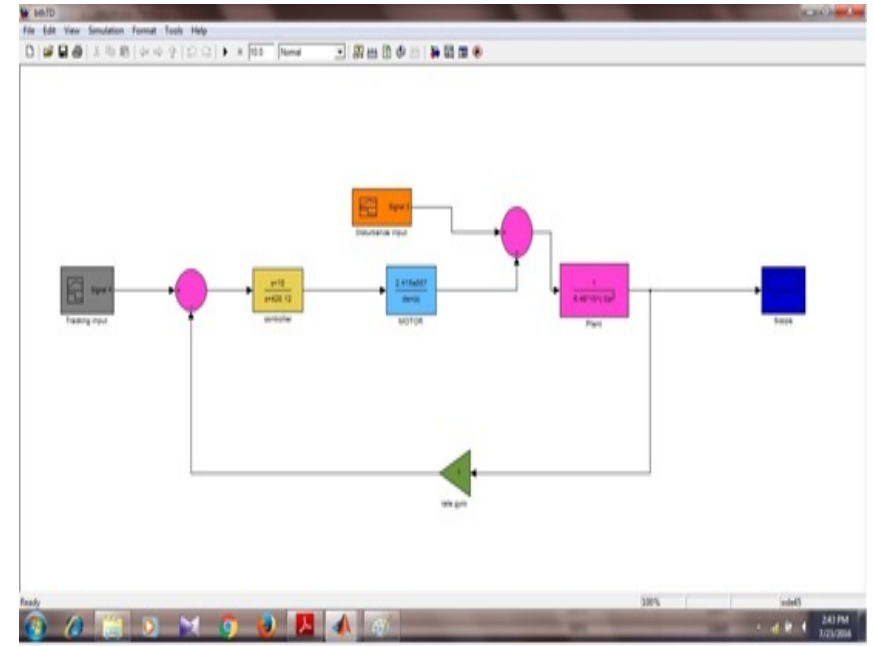

Fig 7 Simulation setup

Simulation outputs for the above three conditions are indicated in Figure 8
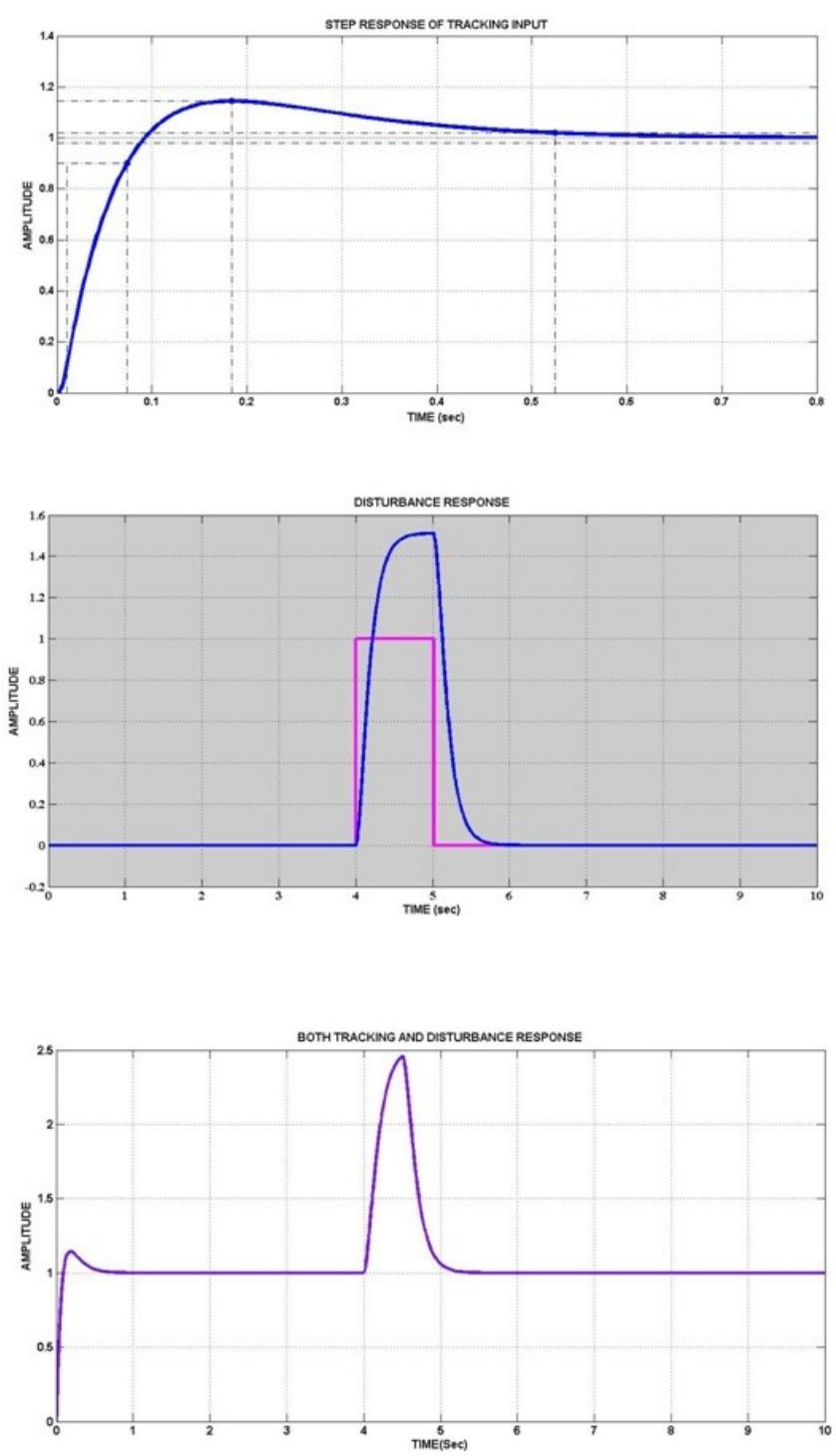

Fig 8: Simulation Results

\section{CONCLUSION}

A Servo system for stabilizing the antenna tracking a moving target has been designed and performance evaluation is obtained by simulation. The disturbance rejection as per the specification has been achieved in addition to tracking. The motor which is of BLDC has been suggested for use in the actual system so also the specification for the Rate Gyro has been worked out for procurement and integration.

\section{ACKNOWLEDGEMENT}

I sincerely thank Defence R\&D Kalyani Center for Technology and Innovation (KCTI) team for providing necessary data, facilities \& time to carry out this task at KCTI Premises, in addition to my regular tasks. Also fruitful suggestions have been accorded by superiors in completing this task in specified time frame, which enabled KCTI to undertake Hardware fabrication to realize this system, based on the design.

\section{REFERENCES}

[1]. Missile Guidance and Control Systems by George M. Siouris

[2]. Missile Guidance and pursuit kinematics, Dynamics and Control by Dr N.A. Shneydor

[3]. Tactical \& strategic missile guidance by Paul Zarchan

[4]. Missile Flight Simulation by Jeffrey Strickland.

\section{BIOGRAPHIES}

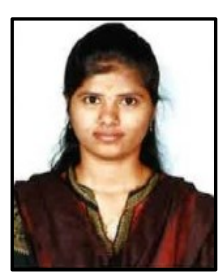

M Geetha Reddy working as Engineer in BFL Defence R\&D KCTI Hyderabad. Pursuing post- graduate in Digital Electronics \& Communication Systems at JIET.

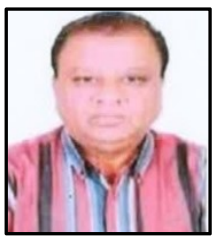

R Sundararajan Sc-'G' (Retd.) from DRDO Hyderabad on super annuation. Worked in areas related to Guidance, Control, and Modelling \& Simulation etc. of Guided weapon system. At present working in BFL Defence R\&D KCTI Hyderabad as Research Director. 